\title{
Constraint Satisfaction over a Non-Boolean Domain: Approximation algorithms and Unique-Games hardness*
}

\author{
Venkatesan Guruswami $^{\dagger} \quad$ Prasad Raghavendra $^{\ddagger}$
}

October 5, 2009

\begin{abstract}
We study the approximability of the MAx $k$-CSP problem over non-boolean domains, more specifically over $\{0,1, \ldots, q-1\}$ for some integer $q$. We extend the techniques of Samorodnitsky and Trevisan [18] to obtain a UGC hardness result when $q$ is a prime. More precisely, assuming the Unique Games Conjecture, we show that it is NP-hard to approximate the problem to a ratio greater than $q^{2} k / q^{k}$. Independent of this work, Austrin and Mossel [2] obtain a more general UGC hardness result using entirely different techniques.

We also obtain an approximation algorithm that achieves a ratio of $C(q) \cdot k / q^{k}$ for some constant $C(q)$ depending only on $q$. Except for constant factors depending on $q$, the algorithm and the UGC hardness result have the same dependence on the arity $k$. It has been pointed out to us [14] that a similar approximation ratio can be obtained by reducing the non-boolean case to a boolean CSP, and appealing to the CMM algorithm [3].

As a subroutine, we design a constant factor(depending on $q$ ) approximation algorithm for the problem of maximizing a semidefinite quadratic form, where the variables are constrained to take values on the corners of the $q$-dimensional simplex. This result generalizes an algorithm of Nesterov [15] for maximizing semidefinite quadratic forms where the variables take $\{-1,1\}$ values.
\end{abstract}

*An extended abstract of this work was presented at APPROX 2008: the 11th International Workshop on Approximation, Randomization and Combinatorial Optimization.

${ }^{\dagger}$ Computer Science Department, Carnegie Mellon University, Pittsburgh, PA 15213. Email: venkatg@cs.cmu.edu. Work done while visiting the School of Mathematics, Institute for Advanced Study, Princeton, NJ. Research supported in part by NSF CCF-0343672 and a David and Lucile Packard Fellowship.

${ }^{\ddagger}$ Microsoft Research New England, Cambridge, MA 02142. Email: prasad@cs.washington.edu. Work done while visiting Princeton University. Research supported in part by NSF CCF-0343672. 


\section{Introduction}

Constraint Satisfaction Problems (CSP) capture a large variety of combinatorial optimization problems that arise in practice. In the MAX $k$-CSP problem, the input consists of a set of variables taking values over a domain(say $\{0,1\}$ ), and a set of constraints with each acting on $k$ of the variables. The objective is to find an assignment of values to the variables that maximizes the number of constraints satisfied. Several classic optimization problems like MAX 3-SAT, MAX CUT fall in to the general framework of CSPs. For most CSPs of interest, the problem of finding the optimal assignment turns out to be NP-hard. To cope with this intractability, the focus shifts to approximation algorithms with provable guarantees. Specifically, an algorithm $\mathcal{A}$ is said to yield an $\alpha$ approximation to a CSP, if on every instance $\Gamma$ of the CSP, the algorithm outputs an assignment that satisfies at least $\alpha$ times as many constraints as the optimal assignment.

Apart from its natural appeal, the study of approximability of MAx $k$-CSP problem is interesting for yet another reason. The best approximation ratio achievable for MAX $k$-CSP equals the optimal soundness of a PCP verifier making at most $k$ queries. In fact, inapproximability results for MAX $k$-CSP have often been accompanied by corresponding developments in analysis of linearity testing.

Over the boolean domain, the problem of MAx $k$-CSP has been studied extensively. For a boolean predicate $P:\{0,1\}^{k} \rightarrow\{0,1\}$, the MAX $k$-CSP $(\mathrm{P})$ problem is the special case of MAX $k$-CSP where all the constraints are of the form $P\left(l_{1}, l_{2}, \ldots, l_{k}\right)$ with each literal $l_{i}$ being either a variable or its negation. For many natural boolean predicates $P$, approximation algorithms and matching NP-hardness results are known for MAx $k$-CSP (P)[10]. For the general MAX $k$-CSP problem over boolean domain, the best known algorithm yields a ratio of $\Omega\left(\frac{k}{2^{k}}\right)$ [3], while any ratio better than $2^{\sqrt{2 k}} / 2^{k}$ is known to be NP-hard to achieve [5]. Further if one assumes the Unique Games Conjecture, then it is NP-hard to approximate MAX $k$-CSP problem to a factor better than $\frac{2 k}{2^{k}}[18]$.

In this work, we study the approximability of the MAx $k$-CSP problem over non-boolean domains, more specifically over $\{0,1, \ldots, q-1\}$ for some integer $q$, obtaining both algorithmic and hardness results (under the UGC) with almost matching approximation factors.

On the hardness side, we extend the techniques of [18] to obtain a UGC hardness result when $q$ is a prime. More precisely, assuming the Unique Games Conjecture, we show that it is NP-hard to approximate the problem to a ratio greater than $q^{2} k / q^{k}$. Except for constant factors depending on $q$, the algorithm and the UGC hardness result have the same dependence on of the arity $k$. Independent of this work, Austrin and Mossel [2] obtain a more general UGC hardness result using entirely different techniques. Technically, our proof extends the Gowers Uniformity based approach of Samorodnitsky and Trevisan [18] to correlations on $q$-ary cubes instead of the binary cube. This is related to the detection of multidimensional arithmetic progressions by a Gowers norm of appropriately large degree. Along the way, we also make a simplification to [18] and avoid the need to obtain a large cross-influence between two functions in a collection with a substantial Uniformity norm; instead our proof works based on large influence of just one function in the collection.

On the algorithmic side, we obtain a approximation algorithm that achieves a ratio of $C(q)$. $k / q^{k}$ for some constant $C(q)$ depending only on $q$. As a subroutine, we design an algorithm for maximizing a positive definite quadratic form with variables forced to take values on the corners of the $q$-dimensional simplex. This is a generalization of an algorithm of Nesterov [15] for maximizing positive definite quadratic form with variables forced to take $\{-1,1\}$ values. This generalization 
could be of independent interest in the design of algorithms for non-boolean CSPs.

Independent of this work, Makarychev and Makarychev [14] brought to our notice a reduction from non-boolean CSPs to the boolean case, which in conjunction with the CMM algorithm [3] yields a better approximation ratio for the MAX $k$-CSP problem. The details of the reduction are described in Section 8. Using the reduction, one can deduce a $q^{2}(1+o(1)) k / q^{k}$ factor UG-hardness for MAX $k$-CSP for arbitrary positive integers $q$, starting from our UG-hardness result for primes $q$.

\subsection{Related Work}

The simplest algorithm for MAX $k$-CSP over boolean domain is to output a random assignment to the variables, thus achieving an approximation ratio of $\frac{1}{2^{k}}$. The first improvement over this trivial algorithm, a ratio of $\frac{2}{2^{k}}$ was obtained by Trevisan [19]. Hast [8] proposed an approximation algorithm with a ratio of $\Omega\left(\frac{k}{\log k 2^{k}}\right)$, which was later improved to the current best known algorithm achieving an approximation factor of $\Omega\left(\frac{k}{2^{k}}\right)[3]$.

On the hardness side, MAX $k$-CSP over the boolean domain was shown to be NP-hard to approximate to a ratio greater than $\Omega\left(2^{2 \sqrt{k}} / 2^{k}\right)$ by Samorodnitsky and Trevisan [17]. The result involved an analysis of a graph-linearity test which was simplified subsequently by Håstad and Wigderson [12]. Later, using the machinery of multi-layered PCP developed in [4], the inapproximability factor was improved to $O\left(2^{\sqrt{2 k}} / 2^{k}\right)$ in [5].

A predicate $P$ is approximation resistant if the best optimal approximation ratio for MAX $k$ CSP $(\mathrm{P})$ is given by the random assignment. While no predicate over 2 variables is approximation resistant, a predicate over 3 variables is approximation resistant if and only if it is implied by the XOR of 3 variables [10, 20]. Almost all predicates on 4 variables were classified with respect to approximation resistance in [9].

In recent years, several inapproximability results for MAx $k$-CSP problems were obtained assuming the Unique Games Conjecture. Firstly, a tight inapproximability of $\Theta\left(\frac{k}{2^{k}}\right)$ was shown in [18]. The proof relies on the analysis of a hypergraph linearity test using the Gowers uniformity norms. Hastad showed that if UGC is true, then as $k$ increases, nearly every predicate $P$ on $k$ variables is approximation resistant [11].

More recently, optimal inapproximability results have been shown for large classes of CSPs assuming the Unique Games Conjecture. Under an additional conjecture, optimal inapproximability results were obtained in [1] for all boolean predicates over 2 variables. Subsequently, it was shown in [16] that for every CSP over an arbitrary finite domain, the best possible approximation ratio is equal to the integrality gap of a well known Semidefinite program. Further the same work also obtains an algorithm that achieves the best possible approximation ratio assuming UGC. Although the results of [16] apply to non-boolean domains, they do not determine the value of the approximation factor explicitly, but only show that it is equal to the integrality gap of an SDP. Further the algorithm proposed in [16] does not yield any approximation guarantee for MAX $k$-CSP unconditionally. Thus neither the inapproximability nor the algorithmic results of this work are subsumed by [16].

Austrin and Mossel [2] obtain a sufficient condition for a predicate $P$ to be approximation resistant. Through this sufficiency condition, they obtain strong UGC hardness results for MAX $k$-CSP problem over the domain $\{1, \ldots, q\}$ for arbitrary $k$ and $q$. For the case when $q$ is a prime power, their results imply a UGC hardness of $k q(q-1) / q^{k}$. The hardness results in this work and 
[2] were obtained independently and use entirely different techniques.

\subsection{Organization of the paper}

We begin with background on the Unique Games conjecture, Gowers norm, and influence of variables in Section 2. In Section 3, we present an efficient algorithm to maximize positive semidefinite forms under additional constraints. This leads to a factor $C(q) k / q^{k}$ approximation algorithm for MAX $k$-CSP over domain size $q$ presented in Section 4 . In Section 5 , we present a linearity test that forms the core of the UGC based hardness reduction. We prove our inapproximability result (for the case when $q$ is a prime) by a reduction from Unique Games in Section 6. The proof uses a technical step bounding a certain expectation by an appropriate Gowers norm; this step is proved in Section 7.

\section{Preliminaries}

For a positive integer $n$, we use the notation $[n]$ for the $\operatorname{ring} \mathbb{Z} /(n)=\{0,1, \ldots, n-1\}$. For a positive integer $q$, let $\Delta_{q}$ denote the following polytope:

$$
\Delta_{q}=\left\{\mathbf{x} \in \mathbb{R}^{q} \quad: \quad x^{(i)} \in\left[-\frac{1}{q-1}, 1\right], \quad \sum_{i \in[q]} x^{(i)}=0\right\}
$$

Let $\operatorname{Vert}\left(\Delta_{q}\right)$ denote the set of $q$ corners(vertices) of the polytope $\Delta_{q}$.

\subsection{Unique Games conjecture}

Definition 2.1. An instance of Unique Games represented as $\Gamma=(\mathcal{X} \cup \mathcal{Y}, E, \Pi,\langle R\rangle)$, consists of a bipartite graph over node sets $\mathcal{X}, \mathcal{Y}$ with the edges $E$ between them. Also part of the instance is a set of labels $\langle R\rangle=\{1, \ldots, R\}$, and a set of permutations $\pi_{v w}:\langle R\rangle \rightarrow\langle R\rangle$ for each edge $e=(v, w) \in E$. An assignment $A$ of labels to vertices is said to satisfy an edge $e=(v, w)$, if $\pi_{v w}(A(v))=A(w)$. The objective is to find an assignment $A$ of labels that satisfies the maximum number of edges.

For sake of convenience, we shall use the following stronger version of Unique Games Conjecture which was shown to be equivalent to the original conjecture [13].

Conjecture 2.2. For all constants $\delta>0$, there exists large enough constant $R$ such that given $a$ bipartite unique games instance $\Gamma=\left(\mathcal{X} \cup \mathcal{Y}, E, \Pi=\left\{\pi_{e}:\langle R\rangle \rightarrow\langle R\rangle: e \in E\right\},\langle R\rangle\right)$ with number of labels $R$, it is NP-hard to distinguish between the following two cases:

- $(1-\delta)$-satisfiable instances: There exists an assignment $A$ of labels such that for $1-\delta$ fraction of vertices $v \in \mathcal{X}$, all the edges $(v, w)$ are satisfied.

- Instances that are not $\delta$-satisfiable: No assignment satisfies more than a $\delta$-fraction of the edges E.

\subsection{Gowers uniformity norm and influence of variables}

We now recall the definition of the Gowers uniformity norm. For an integer $d \geqslant 1$ and a complexvalued function $f: G \rightarrow \mathbb{C}$ defined on an abelian group $G$ (whose group operation we denote by 
+ ), the d'th uniformity norm $U_{d}(f)$ is defined as

$$
U^{d}(f):=\underset{x, y_{1}, y_{2}, \ldots, y_{d}}{\mathbb{E}}\left[\prod_{\substack{S \subseteq\{1,2, \ldots, d\} \\|S| \text { even }}} f\left(x+\sum_{i \in S} y_{i}\right) \prod_{\substack{S \subseteq\{1,2, \ldots, d\} \\|S| \text { odd }}} \overline{f\left(x+\sum_{i \in S} y_{i}\right)}\right] .
$$

where the expectation is taken over uniform and independent choices of $x, y_{0}, \ldots, y_{d-1}$ from the group $G$. Note that $U^{1}(f)=(\underset{x}{\mathbb{E}}[f(x)])^{2}$.

We will be interested in the case when the group $G$ is $[q]^{R}$ for positive integers $q, R$, with group addition being coordinate-wise addition modulo q. $G$ is also closed under coordinate-wise multiplication modulo $q$ by scalars in $[q]$, and thus has a $[q]$-module structure. For technical reasons, we will restrict attention to the case when $q$ is prime and thus our groups will be vector spaces over the field $\mathbb{F}_{q}$ of $q$ elements. For a vector $\mathbf{a} \in[q]^{k}$, we denote by $a_{1}, a_{2}, \ldots, a_{k}$ its $k$ coordinates. We will use 1, $\mathbf{0}$ to denote the all 1's and all 0's vectors respectively (the dimension will be clear from the context). Further denote by $\mathbf{e}_{i}$ the $i^{\text {th }}$ basis vector with 1 in the $i^{\text {th }}$ coordinate and 0 in the remaining coordinates. As we shall mainly be interested in functions over $[q]^{R}$ for a prime $q$, we make our further definitions in this setting. Firstly, every function $f:[q]^{R} \rightarrow \mathbb{C}$ has a Fourier expansion given by:

$$
f(x)=\sum_{\alpha \in[q]^{R}} \hat{f}_{\alpha} \chi_{\alpha}(x)
$$

where $\hat{f}_{\alpha}=\underset{x \in[q]^{R}}{\mathbb{E}}\left[f(x) \chi_{\alpha}(x)\right]$ and $\chi_{\alpha}(x)=\prod_{i=1}^{R} \omega^{\alpha_{i} x_{i}}$ for a $q^{\text {th }}$ root of unity $\omega$.

The central lemma in the hardness reduction relates a large Gowers norm for a function $f$, to the existence of an influential coordinate. Towards this, we define influence of a coordinate for a function over $[q]^{R}$.

Definition 2.3. For a function $f:[q]^{R} \rightarrow \mathbb{C}$ define the influence of the $i^{\text {th }}$ coordinate as follows:

$$
\operatorname{Inf}_{i}(f)=\underset{x}{\mathbb{E}}\left[\operatorname{Var}_{x_{i}}[f]\right] .
$$

The following well known result relates influences to the Fourier spectrum of the function.

Fact. For a function $f:[q]^{R} \rightarrow \mathbb{C}$ and a coordinate $i \in\{1,2, \ldots, R\}$,

$$
\operatorname{Inf}_{i}(f)=\sum_{\alpha_{i} \neq 0, \alpha \in[q]^{R}}\left|\hat{f}_{\alpha}\right|^{2} .
$$

The following lemma is a restatement of Theorem 12 in [18].

Lemma 2.4. There exists an absolute constant $C$ such that, if $f:[q]^{m} \rightarrow \mathbb{C}$ is a function satisfying $|f(x)| \leqslant 1$ for every $x$ then for every $d \geqslant 1$,

$$
U^{d}(f) \leqslant U^{1}(f)+2^{C d} \max _{i} \operatorname{Inf}_{i}(f)
$$




\section{$2.3 \quad$ Noise Operator}

Like many other UGC hardness results, one of the crucial ingredients of our reduction will be a noise operator on functions over $[q]^{R}$. We define the noise operator $T_{1-\varepsilon}$ formally below.

Definition 2.5. For $0 \leqslant \varepsilon \leqslant 1$, define the operator $T_{1-\varepsilon}$ on functions $f:[q]^{R} \rightarrow \mathbb{C}$ as :

$$
T_{1-\varepsilon} f(\mathbf{x})=\underset{\eta}{\mathbb{E}}[f(\mathbf{x}+\eta)]
$$

where each coordinate $\eta_{i}$ of $\eta$ is 0 with probability $1-\varepsilon$ and a random element from $[q]$ with probability $\varepsilon$. The Fourier expansion of $T_{1-\varepsilon} f$ is given by

$$
T_{1-\varepsilon} f(\mathbf{x})=\sum_{\alpha \in[q]^{R}}(1-\varepsilon)^{|\alpha|} \hat{f}_{\alpha} \chi_{\alpha}(x)
$$

Here $|\alpha|$ denotes the number of non-zero coordinates of $\alpha$.

Lemma 2.6. If a function $f:[q]^{R} \rightarrow \mathbb{C}$ satisfies $|f(x)| \leqslant 1$ for all $x$, and $g=T_{1-\varepsilon} f$ then

$$
\sum_{i=1}^{R} \operatorname{Inf}_{i}(g) \leqslant \frac{1}{2 e \ln 1 /(1-\varepsilon)}
$$

Proof. Let $f(x)=\sum_{\alpha} \hat{f}_{\alpha} \chi_{\alpha}(x)$ denote the Fourier expansion of $f$. The function $g$ is given by $g(x)=\sum_{\alpha}(1-\varepsilon)^{|\alpha|} \hat{f}_{\alpha} \chi_{\alpha}(x)$. Hence we get,

$$
\begin{aligned}
\sum_{i=1}^{R} \operatorname{Inf}_{i}(g) & =\sum_{i=1}^{R} \sum_{\alpha, \alpha_{i} \neq 0}(1-\varepsilon)^{2|\alpha|} \hat{f}_{\alpha}^{2}=\sum_{\alpha \in[q]^{R}}(1-\varepsilon)^{2|\alpha|}|\alpha| \hat{f}_{\alpha}^{2} \\
& \leqslant \max _{\alpha \in[q]^{R}}\left((1-\varepsilon)^{2|\alpha|}|\alpha|\right) \cdot \sum_{\alpha} \hat{f}_{\alpha}^{2} \leqslant \max _{\alpha \in[q]^{R}}(1-\varepsilon)^{2|\alpha|}|\alpha|
\end{aligned}
$$

The function $F(x)=x(1-\varepsilon)^{2 x}$ achieves a maximum at $x=-1 / 2 \ln (1-\varepsilon)$. Substituting we get $\sum_{i=1}^{R} \operatorname{Inf}_{i}(g) \leqslant \frac{1}{2 e \ln 1 /(1-\varepsilon)}$.

\section{Positive Definite Quadratic Programs}

In this section, we present an efficient procedure to optimize positive definite quadratic forms with

\begin{tabular}{|c|c|c|}
\hline \multicolumn{3}{|c|}{ QuadraticProgram $\Gamma$} \\
\hline Maximize & $\sum a_{i j}^{(k)(l)} x_{i}^{(k)} \cdot x_{j}^{(l)}$ & \\
\hline Subject to & $\mathbf{x}_{i}=\left(x_{i}^{(0)}, x_{i}^{(1)}, \ldots, x_{i}^{(q-1)}\right) \in \operatorname{Vert}\left(\Delta_{q}\right)$ & $1 \leqslant i \leqslant n$ \\
\hline
\end{tabular}
the variables forced to take values on the $q$-dimensional simplex.

Theorem 3.1. Let $A=\left(a_{i j}^{(k)(l)}\right)$ be a positive definite matrix where $k, l \in[q]$ and $1 \leqslant i, j \leqslant n$. For the quadratic program $\Gamma$, there exists an efficient algorithm that finds an assignment whose value is at least $\frac{2}{\pi(q-1)^{4}}$ of the optimum. 
Proof. Note that $\mathbf{x}_{i} \in \operatorname{Vert}\left(\Delta_{q}\right)$ is equivalent to the following constraints:

$$
\sum_{k \in[q]} x_{i}^{(k)}=0 \quad x_{i}^{(k)} \in\left\{-\frac{1}{q-1}, 1\right\} \quad \forall k \in[q]
$$

For each $i$, eliminate the variable $x_{i}^{(q-1)}$ from the program by substituting $x_{i}^{(q-1)}=-\sum_{j \in[q-1]} x_{i}^{(j)}$. The constraints $\sum_{j} x_{i}^{(j)}=0$ can now be dropped from the quadratic program. Let $\Gamma_{1}$ denote the quadratic program obtained by relaxing every variable $x_{i}^{(j)}$ to take values from the interval $\left[-\frac{1}{q-1}, 1\right]$ instead of $\left\{-\frac{1}{q-1}, 1\right\}$. Clearly, $O P T(\Gamma) \leqslant O P T\left(\Gamma_{1}\right)$. Observe that the objective function is a convex function of the variables $x_{i}^{(j)}$. Hence there is an optimal solution of the program $\Gamma_{1}$ with all the $x_{i}^{(j)} \in\left\{-\frac{1}{q-1}, 1\right\}$ instead of the interval $\left[-\frac{1}{q-1}, 1\right]$. In particular, this implies $O P T\left(\Gamma_{1}\right)=O P T(\Gamma)$.

Let $\Gamma_{2}$ denote the quadratic program obtained by relaxing the range of each variable $x_{i}^{(j)}$ to the interval $[-1,1]$. As the objective value is a convex function of $x_{i}^{(j)}$, there exists optimal solutions to $\Gamma_{2}$ with all variables $x_{i}^{(j)}$ taking values \pm 1 . Relax the range of values $x_{i}^{(j)}$ from $\{-1,1\}$ to unit vectors to obtain a semidefinite program $\Gamma_{3}$. Thus we have $O P T(\Gamma)=O P T\left(\Gamma_{1}\right) \leqslant O P T\left(\Gamma_{2}\right) \leqslant O P T\left(\Gamma_{3}\right)$.

The semidefinite program $\Gamma_{3}$ can be solved efficiently to obtain unit vectors $v_{i}^{(j)}$. Let $O P T\left(\Gamma_{3}\right)$ denote the value of the SDP solution $v_{i}^{(j)}$. Now we use the following result of Nesterov [15] to obtain a solution to $\Gamma_{1}$ from the vectors $v_{i}^{(j)}$.

Theorem 3.2. [15] There exists an efficient procedure that given a positive semidefinite matrix $A=\left(a_{i j}\right)$ and a set of unit vectors $v_{i}$, assigns \pm 1 to the variables $z_{i}$ such that

$$
\sum_{i, j} a_{i j} z_{i} z_{j} \geqslant \frac{2}{\pi} \sum_{i, j} a_{i j}\left\langle v_{i}, v_{j}\right\rangle
$$

Let $z_{i}^{(j)} \in\{-1,1\}$ denote the solution to $\Gamma_{2}$. Define a solution to the quadratic program $\Gamma_{1}$ by scaling the values $z_{i}^{(j)}$ appropriately:

$$
\begin{array}{rlrl}
y_{i}^{(j)} & = & & \forall j \in[q-1] \\
& =-\left(\sum_{j \in[q-1]} z_{i}^{(j)}\right) /(q-1)^{2} & j=q
\end{array}
$$

With $z_{i}^{(j)} \in\{-1,1\}$, it is easy to see that $y_{i}^{(j)} \in\left[-\frac{1}{q-1}, 1\right]$. By definition, we have $\sum_{j} y_{i}^{(j)}=0$. If $\sigma$ denotes the value of the solution $y_{i}^{(j)}$ then,

$$
\sigma \geqslant \frac{2}{\pi(q-1)^{4}} O P T\left(\Gamma_{3}\right) \geqslant \frac{2}{\pi(q-1)^{4}} O P T(\Gamma)
$$

Further since $y_{i}^{(j)}$ form a feasible solution to the quadratic program $\Gamma_{1}$, we have $\sigma \leqslant O P T\left(\Gamma_{1}\right)=$ $O P T(\Gamma)$. Thus $\sigma$ is already a $\frac{2}{\pi(q-1)^{4}}$ approximation for $O P T(\Gamma)$. Towards obtaining an explicit solution to $\Gamma$, define $\mathbf{V}^{0}, \mathbf{V}^{1}, \mathbf{V}^{2}, \ldots, \mathbf{V}^{q-1} \in \mathbb{R}^{q}$ to be the $q$ corners of the polytope $\Delta_{q}$. Since each vector $\mathbf{y}_{i}=\left(y_{i}^{(0)}, y_{i}^{(1)}, \ldots, y_{i}^{(q-1)}\right) \in \Delta_{q}$ it can be expressed as a convex combination of the corners:

$$
\mathbf{y}_{i}=\sum_{j=1}^{q} p_{i}^{(j)} \mathbf{V}^{j}
$$


Round each vector $\mathbf{y}_{i}$ independently to one of the corners $\mathbf{V}^{j}$ using the probability distribution $p_{i}^{(j)}$. Let $\mathbf{x}_{i}$ denote the random variable corresponding to the rounding of vector $\mathbf{y}_{i}$. By definition, we have $\mathbf{E}\left[\mathbf{x}_{i}\right]=\mathbf{y}_{i}$. If $F$ denotes the objective function of the program $\Gamma$, then by convexity of $F$ :

$$
\mathbf{E}\left[F\left(\mathbf{x}_{1}, \mathbf{x}_{2}, \ldots, \mathbf{x}_{n}\right)\right] \geqslant F\left(\mathbf{E}\left[\mathbf{x}_{1}\right], \mathbf{E}\left[\mathbf{x}_{2}\right], \ldots, \mathbf{E}\left[\mathbf{x}_{n}\right]\right) \geqslant \frac{2}{\pi(q-1)^{4}} O P T(\Gamma)
$$

Hence the vectors $\mathbf{x}_{1}, \mathbf{x}_{2}, \ldots, \mathbf{x}_{n}$ form a $\frac{2}{\pi(q-1)^{4}}$-approximate solution to $\Gamma$.

\section{Approximation algorithm for Max $k$-CSP over any domain}

\subsection{Reduction to Max $k$-Equal}

Without loss of generality, we may assume that the $q$-ary domain is $Z_{q}$ the set of integers $\bmod q$. In this section, we shall frequently deal with points in $\mathbb{R}^{q}$. To aid in understanding, we shall always use bold face letters $(\mathbf{x})$ to denote points in $\mathbb{R}^{q}$ and superscripts $\left(x^{(i)}\right)$ to index their coordinates.

Along the lines of $[8,3]$, we first reduce a general MAX $k$-CSP problem to a MAX $k$-EQUAL problem. The MAX $k$-EQUAL problem over a $q$-ary domain is defined as follows:

Definition 4.1 (Max $k$-Equal Problem). Given a set $S$ of clauses of the form $w_{1}-a_{1}=w_{2}-a_{2}=$ $w_{3}-a_{3}=\ldots=w_{k}-a_{k} \bmod q$ with $a_{i} \in Z_{q}$ ( $a_{i}$ can change for different clauses), Find an assignment to the variables $w_{i}$ that maximizes the number of satisfied clauses.

An arbitrary MAX $k$-CSP problem can be reduced to the MAX $k$-EQUAL problem as follows: Let $F\left(w_{1}, \ldots, w_{k}\right)$ denote the constraint in an arbitrary MAx $k$-CSP problem. Let $S(F) \subset[q]^{k}$ denote the set of all satisfying assignments of $F$. For each satisfying assignment $\left(a_{1}, a_{2}, \ldots, a_{k}\right) \in[q]^{k}$, introduce an equality clause $w_{1}-a_{1}=w_{2}-a_{2}=\ldots w_{k}-a_{k} \bmod q$. Clearly the objective value of the MAX $k$-EQUAL instance is at least as much as the original Max $k$-CSP instance. Further the value is at most $q$ times the value of the MAX $k$-CSP instance. Hence a $\gamma$-approximation algorithm for MAX $k$-EQUAL yields a $\gamma / q$ approximation to a general MAX $k$-CSP problem.

\subsection{Quadratic Program}

Let $\mathcal{I}$ be a MAX $k$-EQUAL instance over a set of variables $\mathcal{V}=\left\{w_{1}, \ldots, w_{n}\right\}$ and set of clauses $\mathcal{C}$. Let $O P T(\mathcal{I})$ denote the maximum number of clauses satisfied by any assignment. For each variable $w_{i}$, introduce $q$ variables $x_{i}^{(j)}$ for $j \in[q]$. Let $\mathbf{x}_{i}=\left(x_{i}^{(0)}, x_{i}^{(2)}, \ldots, x_{i}^{(q-1)}\right)$. Consider the following quadratic program in the variables $\mathbf{x}_{i}$.

\begin{tabular}{|c|c|c|}
\hline \multicolumn{3}{|c|}{ QuadraticProgram $\Gamma$} \\
\hline Maximize & $\frac{1}{k^{2}} \sum\left\|\sum x_{i}^{(j)}\right\|^{2}$ & \\
\hline Subject to & $\mathbf{x}_{i}=\left(x_{i}^{(0)}, x_{i}^{(1)}, \ldots, x_{i}^{(q-1)}\right) \in \operatorname{Vert}\left(\Delta_{q}\right)$ & $1 \leqslant i \leqslant n$ \\
\hline
\end{tabular}

In the intended solution, if the variable $w_{i}$ is assigned value $a \in[q]$, then $x_{i}^{(a)}=1$ and $x_{i}^{(j)}=$ $-\frac{1}{q-1}$ for all $j \neq a$. Hence we have $O P T(\Gamma) \geqslant O P T(\mathcal{I})$. 


\subsection{Rounding}

We propose a rounding scheme similar to the one used in [3].

Given a MAX $k$-EQUAL instance $\mathcal{I}=(\mathcal{V}, \mathcal{C})$

- Formulate the quadratic program $\Gamma$, apply Theorem 3.1 to find a solution $\mathbf{x}_{1}, \mathbf{x}_{2}, \ldots, \mathbf{x}_{n}$.

- Set $\delta=\sqrt{\frac{1}{10 k}}$.

- For each variable $w_{i} \in \mathcal{V}$ assign independently

$$
w_{i}=j \quad \text { with probability } \frac{\left(1+\delta x_{i}^{(j)}\right)}{q}, \forall j \in[q]
$$

Theorem 4.2. The approximation algorithm yields an assignment that satisfies $\frac{1}{2 \pi e q(q-1)^{6}} \cdot \frac{k}{q^{k}} O P T$ clauses, where OPT is the number of clauses satisfied by the optimal assignment.

The proof of this theorem is along the lines of [3]. For completeness sake, we present it here.

Proof. By Theorem 3.1, we know that the solution $x_{i}^{j}$ for the quadratic program $\Gamma$ has the objective value at least $\frac{2}{\pi(q-1)^{4}} O P T$. Consider a clause $C \in \mathcal{C}$, given by $w_{1}+a_{1}=w_{2}+a_{2}=\ldots w_{k}+a_{k}$ $\bmod q$. For simplicity of notation, we shall assume $a_{1}=a_{2}=\ldots a_{k}=0$, the general case is very similar. The probability that clause $C$ is satisfied is given by

$$
\operatorname{Pr}[C \text { is satisfied }]=\sum_{j \in[q]} \operatorname{Pr}\left[w_{1}=w_{2}=\ldots w_{k}=j\right]=\sum_{j \in[q]} \prod_{i=1}^{k}\left(\frac{1+\delta x_{i}^{(j)}}{q}\right)
$$

For each $j \in[q]$, define $z_{j}=\left|\left\{i \mid x_{i}^{(j)}=1\right\}\right|$. Rewriting the above equation in terms of $z_{j}$,

$$
\begin{aligned}
\operatorname{Pr}[C \text { is satisfied }]=\frac{1}{q^{k}} \sum_{j \in[q]}(1+\delta)^{z_{j}}\left(1-\frac{\delta}{q-1}\right)^{k-z_{j}} & \geqslant \frac{1}{q^{k}} \sum_{j \in[q]}(1+\delta)^{z_{j}}(1-\delta)^{\frac{k-z_{j}}{q-1}} \\
& =\frac{1}{q^{k}} \sum_{j \in[q]}\left(1-\delta^{2}\right)^{\frac{k-z_{j}}{q-1}}(1+\delta)^{z_{j}-\frac{k-z_{j}}{q-1}}
\end{aligned}
$$

For each $j \in[q]$,

$$
\left(1-\delta^{2}\right)^{\frac{k-z_{j}}{q-1}} \geqslant\left(1-\delta^{2}\right)^{k}=\left(1-\frac{1}{k}\right)^{k} \geqslant\left(1-\frac{1}{k}\right) \frac{1}{e}
$$

Hence,

$$
\operatorname{Pr}[C \text { is satisfied }] \geqslant\left(1-\frac{1}{k}\right) \frac{1}{e} \cdot \frac{1}{q^{k}} \sum_{j \in[q]}(1+\delta)^{z_{j}-\frac{k-z_{j}}{q-1}}
$$

For each $j \in[q]$, define $b_{j}=\sum_{i=1}^{k} x_{i}^{(j)}$. Clearly, $b_{j}=z_{j} \cdot 1+\left(k-z_{i}\right) \cdot\left(-\frac{1}{(q-1)}\right)$. Recall that for each $i$, exactly one of the variables $x_{i}^{(j)}$ is 1 . Hence we have $\sum_{j \in[q]} z_{j}=k$ and $\sum_{j \in[q]} b_{j}=0$.

$$
\operatorname{Pr}[C \text { is satisfied }] \geqslant\left(1-\frac{1}{k}\right) \frac{1}{e} \cdot \frac{1}{q^{k}} \sum_{j \in[q]}(1+\delta)^{b_{j}}
$$


Now we will use the following simple inequality, whose proof is included in the Appendix A.

Lemma 4.3. Let $b_{0}, b_{1}, \ldots, b_{q-1}$ be real numbers such that $\sum_{j \in[q]} b_{j}=0$. For any $\delta>0$,

$$
\sum_{j \in[q]}(1+\delta)^{b_{j}} \geqslant \frac{\delta^{2}}{2 q(q-1)^{2}} \sum_{j \in[q]} b_{j}^{2}
$$

Applying the inequality and using $\left(1-\frac{1}{k}\right) \geqslant \frac{1}{2}$,

$$
\operatorname{Pr}[C \text { is satisfied }] \geqslant \frac{1}{4 e q(q-1)^{2}} \cdot \frac{1}{q^{k}} \delta^{2} \sum_{j \in[q]} b_{j}^{2} \geqslant \frac{1}{4 e q(q-1)^{2}} \cdot \frac{k}{q^{k}} \sum_{j \in[q]} \frac{1}{k^{2}}\left(\sum_{i} x_{i}^{j}\right)^{2}
$$

Using Theorem 3.1, the expected number of clauses satisfied is at least $\frac{1}{2 \pi e q(q-1)^{6}} \cdot \frac{k}{q^{k}} O P T(\Gamma) \geqslant$ $\frac{1}{2 \pi e q(q-1)^{6}} \cdot \frac{k}{q^{k}} O P T(\mathcal{I})$.

\section{$5 \quad$ Linearity Tests and Max $k$-CSP Hardness}

The best approximation ratio possible for MAx $k$-CSP is identical to the best soundness of a $P C P$ verifier for $N P$ that makes $k$ queries. This follows easily by associating the proof locations to CSP variables, and the tests of the verifier to $k$-ary constraints on the locations. In this light, it is natural that the hardness results of $[17,5,18]$ are all associated with a linearity test with a strong soundness. The hardness result in this work is obtained by extending the techniques of [18] from binary to $q$-ary domains. In this section, we describe the test of [18] and outline the extension to it.

For the sake of simplicity, let us consider the case when $k=2^{d}-1$ for some $d$. In [18], the authors propose the following linearity test for functions $F:\{0,1\}^{n} \rightarrow\{0,1\}$.

Complete Hypergraph Test $(F, d)$

- Pick $x_{1}, x_{2}, \ldots, x_{d} \in\{0,1\}^{n}$ uniformly at random.

- Accept if for each $S \subseteq[r]$,

$$
F\left(\sum_{i \in S} x_{i}\right)=\sum_{i \in S} F\left(x_{i}\right)
$$

The test reads the value of the function $F$ at $k=2^{d}-1$ points of a random subspace(spanned by $\left.x_{1}, \ldots, x_{d}\right)$ and checks that $F$ agrees with a linear function on the subspace. Note that a random function $F$ would pass the test with probability $2^{d} / 2^{k}$, since there are $2^{d}$ different satisfying assignments to the $k$ binary values queried by the verifier. The following result is a special case of a more general result by Samorodnitsky and Trevisan [18].

Theorem 5.1. [18] If a function $F:\{0,1\}^{n} \rightarrow\{0,1\}$ passes the Complete Hypergraph Test with probability greater than $2^{d} / 2^{k}+\gamma$, then the function $f(x)=(-1)^{F(x)}$ has a large $d^{\text {th }}$ Gowers norm. Formally, $U^{d}(f) \geqslant C(\gamma, k)$ for some fixed function $C$ of $\gamma, k$.

Towards extending the result to the domain $[q]$, we propose a different linearity test. Again for convenience, let us assume $k=q^{d}$ for some $d$. Given a function $F:[q]^{n} \rightarrow[q]$, the test proceeds as follows: 
Affine Subspace Test $(F, d)$

- Pick $\mathbf{x}, \mathbf{y}_{\mathbf{1}}, \mathbf{y}_{\mathbf{2}}, \ldots, \mathbf{y}_{\mathbf{d}} \in[q]^{n}$ uniformly at random.

- Accept if for each $\mathbf{a} \subseteq[q]^{d}$,

$$
F\left(\mathbf{x}+\sum_{i=1}^{d} a_{i} \mathbf{y}_{\mathbf{i}}\right)=\left(1-\sum_{i=1}^{d} a_{i}\right) F(\mathbf{x})+\sum_{i=1}^{d} a_{i} F\left(\mathbf{x}+\mathbf{y}_{\mathbf{i}}\right)
$$

Essentially, the test queries the values along a randomly chosen affine subspace, and tests if the function $F$ agrees with an affine function on the subspace. Let $\omega$ denote a $q^{\prime}$ th root of unity. From Theorem 7.1 presented in Section 7, the following result can be shown:

Theorem 5.2. If a function $F:[q]^{n} \rightarrow[q]$ passes the Affine Subspace Test with probability greater than $q^{d+1} / q^{k}+\gamma$, then for some $q$ 'th root of unity $\omega \neq 1$, the function $f(x)=\omega^{F(x)}$ has a large $d q$ 'th Gowers norm. Formally, $U^{d q}(f) \geqslant C(\gamma, k)$ for some fixed function $C$ of $\gamma, k$.

The above result follows easily from Theorem 7.1 using techniques of [18], and the proof is ommited here. The Affine Subspace Test forms the core of the UGC based hardness reduction presented in Section 6.

\section{Hardness reduction from Unique Games}

In this section, we will prove a hardness result for approximating MAX $k$-CSP over a domain of size $q$ when $q$ is prime for every $k \geqslant 2$. Let $d$ be such that $q^{d-1}+1 \leqslant k \leqslant q^{d}$. Let us consider the elements of $[q]$ to have a natural order defined by $0<1<\ldots<q-1$. This extends to a lexicographic ordering on vectors in $[q]^{d}$. Denote by $[q]_{<k}^{d}$ the set consisting of the $k$ lexicographically smallest vectors in $[q]^{d}$. We shall identify the set $\{1, \ldots, k\}$ with set of vectors in $[q]_{<k}^{d}$. Specifically, we shall use $\{1, \ldots, k\}$ and vectors in $[q]_{<k}^{d}$ interchangeably as indices to the same set of variables. For a vector $\mathbf{x} \in[q]^{R}$ and a permutation $\pi$ of $\{1, \ldots, R\}$, define $\pi(x) \in[q]^{R}$ defined by $(\pi(x))_{i}=x_{\pi(i)}$.

Let $\Gamma=\left(\mathcal{X} \cup \mathcal{Y}, E, \Pi=\left\{\pi_{e}:\langle R\rangle \rightarrow\langle R\rangle \mid e \in E\right\},\langle R\rangle\right)$ be a bipartite unique games instance. Towards constructing a $k$-CSP instance $\Lambda$ from $\Gamma$, we shall introduce a long code for each vertex in $\mathcal{Y}$. Specifically, the set of variables for the $k$-CSP $\Lambda$ is indexed by $\mathcal{Y} \times[q]^{R}$. Thus a solution to $\Lambda$ consists of a set of functions $F_{w}:[q]^{R} \rightarrow[q]$, one for each $w \in \mathcal{Y}$.

Similar to several other long code based hardness results, we shall assume that the long codes are folded. More precisely, we shall use folding to force the functions $F_{w}$ to satisfy $F_{w}(\mathbf{x}+\mathbf{1})=F(\mathbf{x})+1$ for all $\mathbf{x} \in[q]^{R}$. The $k$-ary constraints in the instance $\Lambda$ are specified by the following verifier. The verifier uses an additional parameter $\varepsilon$ that governs the level of noise in the noise operator. 
- Pick a random vertex $v \in \mathcal{X}$. Pick $k$ vertices $\left\{w_{\mathbf{a}} \mid \mathbf{a} \in[q]_{<k}^{d}\right\}$ from $N(v) \subset \mathcal{Y}$ uniformly at random independently. Let $\pi_{\mathbf{a}}$ denote the permutation on the edge $\left(v, w_{\mathbf{a}}\right)$.

- Sample $\mathbf{x}, \mathbf{y}_{\mathbf{1}}, \mathbf{y}_{\mathbf{2}}, \ldots, \mathbf{y}_{\mathbf{d}} \in[q]^{R}$ uniformly at random. Sample vectors $\eta_{\mathbf{a}} \in[q]^{R}$ for each $\mathbf{a} \in[q]_{<k}^{d}$ from the following distribution: With probability $1-\varepsilon,\left(\eta_{\mathbf{a}}\right)_{j}=0$ and with the remaining probability, $\left(\eta_{\mathbf{a}}\right)_{j}$ is a uniformly random element from $[q]$.

- Query $F_{w_{\mathbf{a}}}\left(\pi_{\mathbf{a}}\left(\mathbf{x}+\sum_{j} a_{j} \mathbf{y}_{\mathbf{j}}+\eta_{\mathbf{a}}\right)\right)$ for each $\mathbf{a} \in[q]_{<k}^{d}$. Accept if the following equality holds for each $\mathbf{a} \in[q]_{<k}^{d}$.

$$
F_{w_{\mathbf{a}}}\left(\pi_{\mathbf{a}}\left(\mathbf{x}+\sum_{j=1}^{d} a_{j} \mathbf{y}_{\mathbf{j}}+\eta_{\mathbf{a}}\right)\right)=\left(1-\sum_{j=1}^{d} a_{j}\right) F_{w_{\mathbf{0}}}\left(\pi_{\mathbf{0}}\left(\mathbf{x}+\eta_{\mathbf{0}}\right)\right)+\sum_{j=1}^{d} a_{j} F_{w_{\mathbf{e}_{\mathbf{j}}}}\left(\pi_{\mathbf{e}_{\mathbf{j}}}\left(\mathbf{x}+\mathbf{y}_{\mathbf{j}}+\eta_{\mathbf{e}_{\mathbf{j}}}\right)\right) .
$$

Theorem 6.1. For all primes $q$, positive integers $d, k$ satisfying $q^{d-1}<k \leqslant q^{d}$, and every $\gamma>0$, there exists small enough $\delta, \varepsilon>0$ such that

- Completeness: If $\Gamma$ is a $(1-\delta)$-satisfiable instance of Unique Games, then there is an assignment to $\Lambda$ that satisfies the verifier's tests with probability at least $(1-\gamma)$

- Soundness: If $\Gamma$ is not $\delta$-satisfiable, then no assignment to $\Lambda$ satisfies the verifier's tests with probability more than $\frac{q^{d+1}}{q^{k}}+\gamma$.

Proof. We begin with the completeness claim, which is straightforward.

Completeness: There exists labelings to the Unique Game instance $\Gamma$ such that for $1-\delta$ fraction of the vertices $v \in \mathcal{X}$ all the edges $(v, w)$ are satisfied. Let $A: \mathcal{X} \cup \mathcal{Y} \rightarrow\langle R\rangle$ denote one such labeling. Define an assignment to the $k$-CSP instance by $F_{w}(\mathbf{x})=x_{A(w)}$ for all $w \in \mathcal{Y}$.

With probability at least $(1-\delta)$, the verifier picks a vertex $v \in \mathcal{X}$ such that the assignment $A$ satisfies all the edges $\left(v, w_{\mathbf{a}}\right)$. In this case for each $\mathbf{a}, \pi_{\mathbf{a}}(A(v))=A\left(w_{\mathbf{a}}\right)$. Let us denote $A(v)=l$. By definition of the functions $F_{w}$, we get $F_{w_{\mathbf{a}}}\left(\pi_{\mathbf{a}}(x)\right)=\left(\pi_{\mathbf{a}}(x)\right)_{A\left(w_{\mathbf{a}}\right)}=x_{\pi_{\mathbf{a}}^{-1}\left(A\left(w_{\mathbf{a}}\right)\right)}=x_{l}$ for all $x \in[q]^{R}$. With probability at least $(1-\varepsilon)^{k}$, each of the vectors $\eta_{\mathbf{a}}$ have their $l^{\text {th }}$ component equal to zero, i.e $\left(\eta_{\mathbf{a}}\right)_{l}=0$. In this case, it is easy to check that all the constraints are satisfied. In conclusion, the verifier accepts the assignment with probability at least $(1-\delta)(1-\varepsilon)^{k}$. For small enough $\delta, \varepsilon$, this quantity is at least $(1-\gamma)$.

Soundness: Suppose there is an assignment given by functions $F_{w}$ for $w \in \mathcal{Y}$ that the verifier accepts with probability greater than $\frac{q^{d+1}}{q^{k}}+\gamma$.

Let $z_{1}, z_{2}, \ldots, z_{k}$ be random variables denoting the $k$ values read by the verifier. Thus $z_{1}, \ldots, z_{k}$ take values in $[q]$. Let $P:[q]^{k} \rightarrow\{0,1\}$ denote the predicate on $k$ variables that represents the acceptance criterion of the verifier. Essentially, the value of the predicate $P\left(z_{1}, \ldots, z_{k}\right)$ is 1 if and only if $z_{1}, \ldots, z_{k}$ values are consistent with some affine function. By definition,

$$
\operatorname{Pr}[\text { Verifier Accepts }]=\underset{v \in \mathcal{X}}{\mathbb{E}} \underset{w_{\mathbf{a}} \in N(v)}{\mathbb{E}} \underset{\mathbf{x}, \mathbf{y} \mathbf{y}_{\mathbf{1}}, \ldots, \mathbf{y}_{\mathbf{d}}}{\mathbb{E}} \underset{\eta_{\mathbf{a}}}{\mathbb{E}}\left[P\left(z_{1}, \ldots, z_{k}\right)\right] \geqslant \frac{q^{d+1}}{q^{k}}+\gamma
$$


Let $\omega$ denote a $q^{t h}$ root of unity. The Fourier expansion of the function $P:[q]^{k} \rightarrow \mathbb{C}$ is given by

$$
P\left(z_{1}, \ldots, z_{k}\right)=\sum_{\alpha \in[q]^{k}} \hat{P}_{\alpha} \chi_{\alpha}\left(z_{1}, \ldots, z_{k}\right)
$$

where $\chi_{\alpha}\left(z_{1}, \ldots, z_{k}\right)=\prod_{i=1}^{k} \omega^{\alpha_{i} z_{i}}$ and $\hat{P}_{\alpha}=\underset{z_{1}, \ldots, z_{k}}{\mathbb{E}}\left[P\left(z_{1}, \ldots, z_{k}\right) \chi_{\alpha}\left(z_{1}, \ldots, z_{k}\right)\right]$. Notice that for $\alpha=\mathbf{0}$, we get $\chi_{\alpha}\left(z_{1}, \ldots, z_{k}\right)=1$. Further,

$$
\hat{P}_{\mathbf{0}}=\underset{z_{1}, \ldots, z_{k}}{\mathbb{E}}\left[P\left(z_{1}, \ldots, z_{k}\right)\right]=\operatorname{Pr}\left[\text { random assignment to } z_{1}, z_{2}, \ldots, z_{k} \text { satisfies } P\right]=\frac{q^{d+1}}{q^{k}}
$$

Substituting the Fourier expansion of $P$, we get

$$
\operatorname{Pr}[\text { Verifier Accepts }]=\frac{q^{d+1}}{q^{k}}+\sum_{\alpha \neq \mathbf{0}} \hat{P}_{\alpha} \underset{v \in \mathcal{X}}{\mathbb{E}} \underset{w_{\mathbf{a}} \in N(v)}{\mathbb{E}} \underset{\mathbf{x}, \mathbf{y}_{\mathbf{1}}, \ldots, \mathbf{y}_{\mathbf{d}}}{\mathbb{E}} \underset{\eta_{\mathbf{a}}}{\mathbb{E}}\left[\chi_{\alpha}\left(z_{1}, \ldots, z_{k}\right)\right]
$$

Recall that the probability of acceptance is greater than $\frac{q^{d+1}}{q^{k}}+\gamma$. Further $\left|\hat{P}_{\alpha}\right| \leqslant 1$ for all $\alpha \in[q]^{k}$. Thus there exists $\alpha \neq 0$ such that,

$$
\left|\underset{v \in \mathcal{X}}{\mathbb{E}} \underset{w_{\mathbf{a}} \in N(v)}{\mathbb{E}, \mathbf{y} \mathbf{1}, \ldots, \mathbf{y}_{\mathbf{d}}} \underset{\eta_{\mathbf{a}}}{\mathbb{E}}\left[\chi_{\alpha}\left(z_{1}, \ldots, z_{k}\right)\right]\right| \geqslant \frac{\gamma}{q^{k}}
$$

For each $w \in \mathcal{Y}, t \in[q]$, define the function $f_{w}^{(t)}:[q]^{d} \rightarrow \mathbb{C}$ as $f_{w}^{(t)}(x)=\omega^{t F_{w}(x)}$. For convenience we shall index the vector $\alpha$ with the set $[q]_{<k}^{d}$ instead of $\{1, \ldots, k\}$. In this notation,

$$
\left|\underset{v \in \mathcal{X}}{\mathbb{E}} \underset{w_{\mathbf{a}} \in N(v)}{\mathbb{E}} \underset{\mathbf{x}, \mathbf{y}_{\mathbf{1}}, \ldots, \mathbf{y}_{\mathbf{d}}}{\mathbb{E}} \underset{\eta_{\mathbf{a}}}{\mathbb{E}}\left[\prod_{\mathbf{a} \in[q]_{<k}^{d}} f_{w_{\mathbf{a}}}^{\left(\alpha_{\mathbf{a}}\right)}\left(\pi_{\mathbf{a}}\left(\mathbf{x}+\sum_{i=1}^{d} a_{i} \mathbf{y}_{\mathbf{i}}+\eta_{\mathbf{a}}\right)\right)\right]\right| \geqslant \frac{\gamma}{q^{k}}
$$

Let $g_{w}^{(t)}:[q]^{d} \rightarrow \mathbb{C}$ denote the smoothened version of function $f_{w}^{(t)}$. Specifically, let $g_{w}^{(t)}(x)=$ $T_{1-\varepsilon} f_{w}^{(t)}(x)=\mathbf{E}_{\eta}\left[f_{w}^{(t)}(x+\eta)\right]$ where $\eta$ is generated from $\varepsilon$-noise distribution. Since each $\eta_{\mathbf{a}}$ is independently chosen, we can rewrite the above expression,

$$
\left|\underset{v \in \mathcal{X}}{\mathbb{E}} \underset{w_{\mathbf{a}} \in N(v)}{\mathbb{E}} \underset{\mathbf{x}, \mathbf{y}_{\mathbf{1}}, \ldots, \mathbf{y}_{\mathbf{d}}}{\mathbb{E}}\left[\prod_{\mathbf{a} \in[q]_{<k}^{d}} g_{w_{\mathbf{a}}}^{\left(\alpha_{\mathbf{a}}\right)}\left(\pi_{\mathbf{a}}\left(\mathbf{x}+\sum_{i=1}^{d} a_{i} \mathbf{y}_{\mathbf{i}}\right)\right)\right]\right| \geqslant \frac{\gamma}{q^{k}}
$$

For each $v \in \mathcal{X}, t \in[q]$, define the function $g_{v}^{(t)}:[q]^{d} \rightarrow \mathbb{C}$ as $g_{v}^{(t)}(x)=\mathbf{E}_{w \in N(v)}\left[g_{w}^{(t)}\left(\pi_{v w}(x)\right)\right]$. As the vertices $w_{\mathbf{a}}$ are chosen independent of each other,

$$
\left|\underset{v \in \mathcal{X}}{\mathbb{E} \mathbf{x}, \mathbf{y} \mathbf{1}, \ldots, \mathbf{y}_{\mathbf{d}}} \underset{\mathbf{a} \in[q]_{<k}^{d}}{\mathbb{E}}\left[g_{v}^{\left(\alpha_{\mathbf{a}}\right)}\left(\mathbf{x}+\sum_{i=1}^{d} a_{i} \mathbf{y}_{\mathbf{i}}\right)\right]\right| \geqslant \frac{\gamma}{q^{k}}
$$

As $\alpha \neq 0$, there exists an index $\mathbf{b} \in[q]_{<k}^{d}$ such that $\alpha_{\mathbf{b}} \neq 0$. For convenience let us denote $c=\alpha_{\mathbf{b}}$. Define

$$
\kappa=2^{-C d q}\left(\frac{\gamma}{2 q^{k}}\right)^{2^{d q}}
$$


where $C$ is the absolute constant defined in Lemma 2.4.

For each $v \in \mathcal{X}$, define the set of labels $L(v)=\left\{i \in\langle R\rangle: \operatorname{Inf}_{i}\left(g_{v}^{c}\right) \geqslant \kappa\right\}$. Similarly for each $w \in \mathcal{Y}$, let $L(w)=\left\{i \in\langle R\rangle: \operatorname{Inf}_{i}\left(g_{w}^{c}\right) \geqslant \kappa / 2\right\}$. Obtain a labeling $A$ to the Unique Games instance $\Gamma$ as follows : For each vertex $u \in \mathcal{X} \cup \mathcal{Y}$, if $L(u) \neq \phi$ then assign a randomly chosen label from $L(u)$, else assign a uniformly random label from $\langle R\rangle$.

The functions $g_{w}^{(c)}$ are given by $g_{w}^{(c)}=T_{1-\varepsilon} f_{w}^{(c)}$ where $f_{w}^{(c)}$ is bounded in absolute value by 1 . By Lemma 2.6, therefore, the sum of its influences is bounded by $\frac{1}{e \ln 1 /(1-\varepsilon)}$. Consequently, for all $w \in \mathcal{Y}$ the size of the label set $L(w)$ is bounded by $\frac{2}{\kappa e \ln 1 /(1-\varepsilon)}$. Applying a similar argument to $v \in \mathcal{X},|L(v)| \leqslant \frac{1}{\kappa e \ln 1 /(1-\varepsilon)}$.

For at least $\gamma / 2 q^{k}$ fraction of vertices $v \in \mathcal{X}$ we have,

$$
\left|\underset{\mathbf{x}, \mathbf{y}_{\mathbf{1}}, \ldots, \mathbf{y}_{\mathbf{d}}}{\mathbb{E}}\left[\prod_{\mathbf{a} \in[q]_{<k}^{d}} g_{v}^{\left(\alpha_{\mathbf{a}}\right)}\left(\mathbf{x}+\sum_{i=1}^{d} a_{i} \mathbf{y}_{\mathbf{i}}\right)\right]\right| \geqslant \frac{\gamma}{2 q^{k}}
$$

We shall refer to these vertices as good vertices. Fix a good vertex $v$.

Observe that for each $u \in \mathcal{X} \cup \mathcal{Y}$ the functions $g_{u}^{(t)}$ satisfy $\left|g_{u}^{(t)}(x)\right| \leqslant 1$ for all $x$. Now we shall apply Theorem 7.1 to conclude that the functions $g_{v}^{(t)}$ have a large Gowers norm. Specifically, consider the collection of functions given by $f_{\mathbf{a}}=g_{v}^{\left(\alpha_{\mathbf{a}}\right)}$ for $\mathbf{a} \in[q]_{<k}^{d}$, and $f_{\mathbf{a}}=1$ for all $\mathbf{a} \notin[q]_{<k}^{d}$. From Theorem 7.1, we get

$$
\min _{\mathbf{a}} U^{d q}\left(g_{v}^{\left(\alpha_{\mathbf{a}}\right)}\right) \geqslant\left(\frac{\gamma}{2 q^{k}}\right)^{2^{d q}}
$$

In particular, this implies $U^{d q}\left(g_{v}^{(c)}\right) \geqslant\left(\frac{\gamma}{2 q^{k}}\right)^{2^{d q}}$. Now we shall use Lemma 2.4 to conclude that the function $g_{v}$ has influential coordinates. Towards this, observe that the functions $f_{w}^{(t)}$ satisfy $f_{w}^{(t)}(x+1)=f_{w}^{(t)}(x) \cdot \omega^{t}$ due to folding. Thus for all $t \neq 0$ and all $w \in \mathcal{Y}, \mathbf{E}_{x}\left[f_{w}^{(t)}(x)\right]=0$. Specifically for $c \neq 0$,

$$
U^{1}\left(g_{v}^{(c)}\right)=\left(\underset{x}{\mathbb{E}}\left[g_{v}^{(c)}(x)\right]\right)^{2}=\left(\underset{w \in N(v)}{\mathbb{E}} \underset{\eta}{\mathbb{E}} \underset{x}{\mathbb{E}}\left[f_{w}^{(c)}(x+\eta)\right]\right)^{2}=0
$$

Hence it follows from Lemma 2.4 that there exists influential coordinates $i$ with $\operatorname{Inf}_{i}\left(g_{v}^{(c)}\right) \geqslant$ $2^{-C d q}\left(\frac{\gamma}{2 q^{k}}\right)^{2^{d q}}=\kappa$. In other words, $L(v)$ is non-empty. Observe that, due to convexity of influences,

$$
\operatorname{Inf}_{i}\left(g_{v}^{(c)}\right)=\operatorname{Inf}_{i}\left(\underset{w \in N(v)}{\mathbb{E}}\left[g_{w}^{(c)}\right]\right) \leqslant \underset{w \in N(v)}{\mathbb{E}} \operatorname{Inf}_{\pi_{v w}(i)}\left(\left[g_{w}^{(c)}(x)\right]\right)
$$

If the coordinate $i$ has influence at least $\kappa$ on $g_{v}^{(c)}$, then the coordinate $\pi_{v w}(i)$ has an influence of at least $\kappa / 2$ for at least $\kappa / 2$ fraction of neighbors $w \in N(v)$. The edge $\pi_{v w}$ is satisfied if $i$ is assigned to $v$, and $\pi_{w v}(i)$ is assigned to $w$. This event happens with probability at least $\frac{1}{|L(u)||L(v)|} \geqslant(e \kappa \ln 1 /(1-\varepsilon))^{2} / 2$ for at least $\kappa / 2$ fraction of the neighbors $w \in N(v)$. As there are at least $\left(\gamma / 2 q^{k}\right)$ fraction of good vertices $v$, the assignment satisfies at least $\left(\gamma / 2 q^{k}\right)(e \kappa \ln 1 /(1-\varepsilon))^{2} \kappa / 4$ fraction of the unique games constraints. By choosing $\delta$ smaller than this fraction, the proof is complete. 
Since each test performed by the verifier involve $k$ variables, by the standard connection between hardness of MAx $k$-CSP and $k$-query PCP verifiers, we get the following hardness result conditioned on the UGC.

Corollary 6.2. Assuming the Unique Games conjecture, for every prime $q$, it is NP-hard to approximate MAX $k$-CSP over domain size $q$ within a factor that is greater than $q^{2} k / q^{k}$.

Using the reduction of [14], the above UG-hardness result can be extended from primes to arbitrary composite number $q$.

Corollary 6.3. [14] Assuming the Unique Games conjecture, for every positive integer q, it is $N P$-hard to approximate MAX $k$-CSP over domain size $q$ within a factor that is greater than $q^{2} k(1+o(1)) / q^{k}$.

\section{Gowers Norm and Multidimensional Arithmetic Progressions}

The following theorem forms a crucial ingredient in the soundness analysis in the proof of Theorem 6.1 .

Theorem 7.1. Let $q \geqslant 2$ be a prime and $G$ be a $\mathbb{F}_{q}$-vector space. Then for all positive integers $\ell \leqslant q$ and $d$, and all collections $\left\{f_{\mathbf{a}}: G \rightarrow \mathbb{C}\right\}_{\mathbf{a} \in[\ell]^{d}}$ of $\ell^{d}$ functions satisfying $\left|f_{\mathbf{a}}(x)\right| \leqslant 1$ for every $x \in G$ and $\mathbf{a} \in[\ell]^{d}$, the following holds:

$$
\left|\underset{x, y_{1}, y_{2}, \ldots, y_{d}}{\mathbb{E}}\left[\prod_{\mathbf{a} \in[\ell]^{d}} f_{\mathbf{a}}\left(x+a_{1} y_{1}+a_{2} y_{2}+\cdots+a_{d} y_{d}\right)\right]\right| \leqslant \min _{\mathbf{a} \in[\ell]^{d}}\left(U^{d \ell}\left(f_{\mathbf{a}}\right)\right)^{1 / 2^{d \ell}}
$$

The proof of the above theorem is via double induction on $d, \ell$. We first prove the theorem for the one-dimensional case, i.e., $d=1$ and every $\ell, 1 \leqslant \ell<q$ (Lemma 7.3). This will be done through induction on $\ell$. We will then prove the result for arbitrary $d$ by induction on $d$.

Remark 7.2. Green and Tao, in their work [7] on configurations in the primes, isolate and define a property of a system of linear forms that ensures that the degree $t$ Gowers norm is sufficient to analyze patterns corresponding to those linear forms, and called this property complexity (see Definition 1.5 in [7]). Gowers and Wolf [6] later coined the term Cauchy-Schwartz (CS) complexity to refer to this notion of complexity. For example, the CS-complexity of the $q$ linear forms $x, x+$ $y, x+2 y, \ldots, x+(q-1) y$ corresponding to a $q$-term arithmetic progression equals $q-2$, and the $U^{q-1}$ norm suffices to analyze them. It can similarly be shown that the CS-complexity of the $d$-dimensional arithmetic progression (with $q^{d}$ linear forms as in $(3)$ ) is at most $d(q-1)-1$. In our application, we need a "multi-function" version of these statements, since we have a different function $f_{\mathbf{a}}$ for each linear form $x+\mathbf{a} \cdot \mathbf{y}$. We therefore work out a self-contained proof of Theorem 7.1 in this setting.

Lemma 7.3. Let $q \geqslant 2$ be prime and $\ell, 1 \leqslant \ell \leqslant q$, be an integer, and $G$ be a $\mathbb{F}_{q}$-vector space. Let $\left\{h_{\alpha}: G \rightarrow \mathbb{C}\right\}_{\alpha \in[\ell]}$ be a collection of $\ell$ functions such that $\left|h_{\alpha}(x)\right| \leqslant 1$ for all $\alpha \in[\ell]$ and $x \in G$. Then

$$
\left|\underset{x, y_{1}}{\mathbb{E}}\left[\prod_{\alpha \in[\ell]} h_{\alpha}\left(x+\alpha y_{1}\right)\right]\right| \leqslant \min _{\alpha \in[\ell]}\left(U^{\ell}\left(h_{\alpha}\right)\right)^{\frac{1}{2^{\ell}}} .
$$


Proof. The proof is by induction on $\ell$. For $\ell=1$, the LHS of (4) equals $\underset{x}{\mathbb{E}}\left[h_{0}(x)\right] \mid$, and the RHS equals $\sqrt{U^{1}\left(h_{0}\right)}$. By definition $U^{1}\left(h_{0}\right)=\underset{x, y_{1}}{\mathbb{E}}\left[h_{0}(x) \overline{h_{0}\left(x+y_{1}\right)}\right]=\left|\underset{x}{\mathbb{E}}\left[h_{0}(x)\right]\right|^{2}$.

Now consider $\ell$ satisfying $1<\ell \leqslant q$. By a change of variables it suffices to upper bound the LHS of (4) by $\left(U^{\ell}\left(h_{\ell-1}\right)\right)^{1 / 2^{\ell}}$. We have

$$
\begin{aligned}
\left|\underset{x, y_{1}}{\mathbb{E}}\left[\prod_{\alpha \in[\ell]} h_{\alpha}\left(x+\alpha y_{1}\right)\right]\right|^{2} & \leqslant \underset{x}{\mathbb{E}}\left[\left|h_{0}(x)\right|^{2}\right] \cdot \underset{x}{\mathbb{E}}\left[\left|\underset{y_{1}^{\prime}}{\mathbb{E}}\left[\prod_{\alpha \in\{1, \ldots, \ell-1\}} h_{\alpha}\left(x+\alpha y_{1}^{\prime}\right)\right]\right|^{2}\right] \\
& \leqslant \underset{x, y_{1}^{\prime}, z_{1}^{\prime}}{\mathbb{E}}\left[\prod_{\alpha \in\{1, \ldots, \ell-1\}} h_{\alpha}\left(x+\alpha y_{1}^{\prime}\right) \overline{h_{\alpha}\left(x+\alpha z_{1}^{\prime}\right)}\right] \\
& =\underset{x, y_{1}, z_{1}}{\mathbb{E}}\left[\prod_{\alpha \in\{0,1, \ldots, \ell-2\}} h_{\alpha+1}\left(x+\alpha y_{1}\right) \overline{h_{\alpha+1}\left(x+\alpha y_{1}+(\alpha+1) z_{1}\right)}\right] \\
& =\underset{z_{1}}{\mathbb{E}}\left[\underset{x, y_{1}}{\mathbb{E}}\left[\prod_{\alpha \in[\ell-1]} \tilde{h}_{\alpha}^{z_{1}}\left(x+\alpha y_{1}\right)\right]\right]
\end{aligned}
$$

where we define $\tilde{h}_{\alpha}^{z_{1}}(t):=h_{\alpha+1}(t) \overline{h_{\alpha+1}\left(t+(\alpha+1) z_{1}\right)}$. By induction hypothesis, the inner expectation in (5) satisfies

$$
\underset{x, y_{1}}{\mathbb{E}}\left[\prod_{\alpha \in[\ell-1]} \tilde{h}_{\alpha}^{z_{1}}\left(x+\alpha y_{1}\right)\right] \leqslant\left(U^{\ell-1}\left(\tilde{h}_{\ell-2}^{z_{1}}\right)\right)^{\frac{1}{2^{\ell-1}}} .
$$

Now,

$$
\begin{aligned}
\left(\underset{z_{1}}{\mathbb{E}}\left[U^{\ell-1}\left(\tilde{h}_{\ell-2}^{z_{1}}\right)^{\frac{1}{2^{\ell-1}}}\right]\right)^{2^{\ell-1}} & \leqslant \underset{z_{1}}{\mathbb{E}}\left[U^{\ell-1}\left(\tilde{h}_{\ell-2}^{z_{1}}\right)\right] \\
& =\underset{z_{1}}{\mathbb{E}} \underset{x, z_{2}, \ldots, z_{\ell}}{\mathbb{E}}\left[\prod_{\substack{S \subseteq\{2,3, \ldots, d\} \\
|S| \text { even }}} h_{h_{\ell-1}\left(x+\sum_{i \in S} z_{i}\right) \overline{h_{\ell-1}\left(x+(\ell-1) z_{1}+\sum_{i \in S} z_{i}\right)}}\right. \\
& \left.\prod_{\substack{S \subseteq\{2,3, \ldots, d\} \\
|S| \text { odd }}} \overline{h_{\ell-1}\left(x+\sum_{i \in S} z_{i}\right)} h_{\ell-1}\left(x+(\ell-1) z_{1}+\sum_{i \in S} z_{i}\right)\right]
\end{aligned}
$$

where the last step uses the fact that for a random choice of $z_{1},(\ell-1) z_{1}$ is distributed uniformly in $G$ (this is why we need $q$ to be a prime). Combining (5), (6), and (7), we obtain our desired conclusion $\left|\underset{x, y_{1}}{\mathbb{E}}\left[\prod_{\alpha \in[\ell]} h_{\alpha}\left(x+\alpha y_{1}\right)\right]\right| \leqslant\left(U^{\ell}\left(h_{\ell-1}\right)\right)^{1 / 2^{\ell}}$.

Proof of Theorem 7.1: Fix an arbitrary $\ell, 1 \leqslant \ell \leqslant q$. We will prove the result by induction on $d$. The base case $d=1$ is the content of Lemma 7.3, so it remains to consider the case $d>1$.

By a change of variables, it suffices to upper bound the LHS of $(3)$ by $\left(U^{d \ell}\left(f_{(\ell-1) 1}\right)\right)^{1 / 2^{d \ell}}$, and this is what we will prove. 
For $\alpha \in[\ell]$, and $y_{2}, y_{3}, \ldots, y_{d} \in G$, define the function

$$
g_{\alpha}^{y_{2}, \ldots, y_{d}}(x)=\prod_{\left.\mathbf{b}=\left(b_{2}, b_{3}, \ldots, b_{d}\right) \in[\ell]\right]^{d-1}} f_{(\alpha, \mathbf{b})}\left(x+b_{2} y_{2}+\cdots+b_{d} y_{d}\right)
$$

The LHS of (3), raised to the power $2^{d \ell}$, equals

$$
\begin{aligned}
\left|\underset{y_{2}, \ldots, y_{d}}{\mathbb{E}} \underset{x, y_{1}}{\mathbb{E}}\left[\prod_{\alpha \in[\ell]} g_{\alpha}^{y_{2}, \ldots, y_{d}}\left(x+\alpha y_{1}\right)\right]\right|^{2^{d \ell}} & \leqslant\left(\underset{y_{2}, \ldots, y_{d}}{\mathbb{E}}\left[\left|\underset{x, y_{1}}{\mathbb{E}} \prod_{\alpha \in[\ell]} g_{\alpha}^{y_{2}, \ldots, y_{d}}\left(x+\alpha y_{1}\right)\right|^{2^{\ell}}\right]\right)^{2^{(d-1) \ell}} \\
& \leqslant\left|\underset{y_{2}, \ldots, y_{d}}{\mathbb{E}} U^{\ell}\left(g_{\ell-1}^{y_{2}, \ldots, y_{d}}\right)\right|^{2^{(d-1) \ell}} \quad \text { (using Lemma 7.3) } \\
& =\left|\underset{y_{2}, \ldots, y_{d}}{\mathbb{E}} \underset{x, z_{1}, \ldots, z_{\ell}}{\mathbb{E}}\left[\prod_{S \subseteq\{1,2, \ldots, \ell\}} g_{\ell-1}^{y_{2}, \ldots, y_{d}}\left(x+\sum_{i \in S} z_{i}\right)\right]\right|^{2^{(d-1) \ell}}
\end{aligned}
$$

Defining the function

$$
H_{\mathbf{b}}^{z_{1}, \ldots, z_{\ell}}(t):=\prod_{S \subseteq\{1,2, \ldots, \ell\}} f_{(\ell-1, \mathbf{b})}\left(t+\sum_{i \in S} z_{i}\right)
$$

for every $\mathbf{b} \in[\ell]^{d-1}$ and $z_{1}, \ldots, z_{\ell} \in G$, the last expression equals

$$
\left.\mid \underset{z_{1}, \ldots, z_{\ell} x, y_{2}, \ldots, y_{d}}{\mathbb{E}} \underset{\left.\mathbf{b}=\left(b_{2}, \ldots, b_{d}\right) \in[\ell]\right]^{d-1}}{\mathbb{E}} H_{\mathbf{b}}^{z_{1}, \ldots, z_{\ell}}\left(x+b_{2} y_{2}+\cdots+b_{d} y_{d}\right)\right]\left.\right|^{2^{(d-1) \ell}}
$$

which is at most

$$
\underset{z_{1}, \ldots, z_{\ell}}{\mathbb{E}}\left[\left|\underset{x, y_{2}, \ldots, y_{d}}{\mathbb{E}}\left[\prod_{\left.\mathbf{b}=\left(b_{2}, \ldots, b_{d}\right) \in[\ell]\right]^{d-1}} H_{\mathbf{b}}^{z_{1}, \ldots, z_{\ell}}\left(x+b_{2} y_{2}+\cdots+b_{d} y_{d}\right)\right]\right|^{2^{(d-1) \ell}}\right]
$$

By the induction hypothesis, (10) is at most

$$
\underset{z_{1}, \ldots, z_{\ell}}{\mathbb{E}}\left[U^{(d-1) \ell}\left(H_{(\ell-1) 1}^{z_{1}, \ldots, z_{\ell}}\right)\right]
$$

Recalling the definition of $H_{\mathbf{b}}^{z_{1}, \ldots, z_{\ell}}$ from (9), the above expectation equals

$$
\underset{z_{1}, \ldots, z_{\ell}}{\mathbb{E}} \underset{\substack{x,\left\{z_{j}^{\prime}\right\} \\ 1 \leqslant j \leqslant(d-1) \ell}}{\mathbb{E}}\left[\prod_{\substack{S \subseteq\{1,2, \ldots, \ell\} \\ T \subseteq\{1,2, \ldots,(d-1) \ell\}}} f_{(\ell-1) \mathbf{1}}\left(x+\sum_{i \in S} z_{i}+\sum_{j \in T} z_{j}^{\prime}\right)\right]
$$

which clearly equals $U^{d \ell}\left(f_{(\ell-1) \mathbf{1}}\right)$. 


\section{Extending the CMM Algorithm for Non-Boolean CSPs}

In this section, we outline a reduction from Non-boolean CSPs to boolean CSPs which in conjunction with CMM algorithm yields another algorithm for MAX $k$-CSP.

Consider the case when $q=2^{t}$ for an integer $t$. Given a CSP $\mathcal{I}=(\mathcal{V}, \mathcal{C})$, for each variable $x \in \mathcal{V}$ in the $q$-ary CSP, introduce $t$ boolean variables corresponding to the encoding of the value $x$ in to binary. Every constraint on $k$ variables in $\mathcal{V}$, translate in to a constraint on $k t$ of the boolean variables. Using the CMM algorithm on the boolean CSP instance, obtain an assignment of value at least $0.44 \frac{k t}{2^{k t}} O P T(\mathcal{I})>0.44 \frac{k}{q^{k}} O P T(\mathcal{I})$.

Set $r=2^{\lfloor\log q\rfloor}$. Then we have $q \geqslant r>q / 2$. We will reduce the $q$-ary CSP instance $\mathcal{I}=(\mathcal{V}, \mathcal{C})$ to a CSP $\mathcal{I}^{\prime}=\left(\mathcal{V}^{\prime}, \mathcal{C}^{\prime}\right)$ over a domain of size $r$. The variables of $\mathcal{I}^{\prime}$ are the same as that of $\mathcal{I}$, i.e $\mathcal{V}=\mathcal{V}^{\prime}$. For each variable $x \in \mathcal{V}^{\prime}$, its domain(set of allowed values) is a randomly chosen subset of size $r$ of the set $[q]$. Thus the domain of each variable in $\mathcal{V}^{\prime}$ is a set of size $r$. The constraints in $\mathcal{C}^{\prime}$ are the same as the constraints in $\mathcal{C}$ in the following sense: For any constraint $c \in \mathcal{C}$, there is a corresponding constraint $c^{\prime} \in \mathcal{C}^{\prime}$ whose satisfying assignments are the same as that of $c$.

Consider the optimal assignment $\mathcal{A}$ to the instance $\mathcal{I}$. We will obtain an assignment to the instance $\mathcal{I}^{\prime}$ as follows: For a variable $x \in \mathcal{V}$, if the assignment $\mathcal{A}(x)$, is not an allowed value for $x$ in $\mathcal{I}^{\prime}$, just reassign a random allowed value to $x$. For any given $k$-tuple of variables $\left(x_{1}, \ldots, x_{k}\right)$, with probability at least $(r / q)^{k}$ over the choice of the random restriction, all the values $\mathcal{A}\left(x_{1}\right), \mathcal{A}\left(x_{2}\right), \ldots, \mathcal{A}\left(x_{k}\right)$ are allowed. Thus the expected number of constraints $\mathcal{C}^{\prime}$ satisfied by the optimal assignment $\mathcal{A}$ is at least $\left(\frac{r}{q}\right)^{k} \cdot O P T(\mathcal{I})$. Running the CMM algorithm on the instance $\mathcal{I}^{\prime}$ produces an assignment that satisfies at least $0.44 \frac{k}{r^{k}} \cdot\left(\frac{r}{q}\right)^{k} \cdot O P T(\mathcal{I}) \geqslant 0.44 \frac{k}{q^{k}} \cdot O P T(\mathcal{I})$.

\section{References}

[1] P. Austrin. Towards sharp inapproximability for any 2-csp. In FOCS : IEEE Symposium on Foundations of Computer Science, 307-317, 2007. 3

[2] P. Austrin and E. Mossel. Approximation resistant predicates from pairwise independence. Electronic Colloqium on Computational Complexity, TR08-009, 2008. 1, 2, 3, 4

[3] M. Charikar, K. Makarychev, and Y. Makarychev. Near-optimal algorithms for maximum constraint satisfaction problems. In Proceedings of the 18th Annual ACM-SIAM Symposium on Discrete Algorithms, pages 62-68, 2007. 1, 2, 3, 8, 9

[4] I. Dinur, V. Guruswami, S. Khot, and O. Regev. A new multilayered PCP and the hardness of hypergraph vertex cover. SIAM J. Computing, 34(5):1129-1146, 2005. 3

[5] L. Engebretsen and J. Holmerin. More efficient queries in PCPs for NP and improved approximation hardness of maximum CSP. In STACS, volume 3404 of Lecture Notes in Computer Science, pages 194-205, 2005. 2, 3, 10

[6] W. T. Gowers and J. Wolf. The true complexity of a system of linear equations. arXiv:math.NT/0711.0185, 2007. 15

[7] B. Green and T. Tao. Linear equations in primes. arXiv:math.NT/0606088v1, 2006. 15 
[8] G. Hast. Approximating MAX kCSP - outperforming a random assignment with almost a linear factor. In ICALP: Annual International Colloquium on Automata, Languages and Programming, pages 956-968, 2005. 3, 8

[9] G. Hast. Beating a random assignment - approximating constraint satisfaction problems. Phd Thesis, Royal Institute of Technology 2005. 3

[10] J. Håstad. Some optimal inapproximability results. Journal of the ACM, 48(4):798-859, 2001. 2,3

[11] J. Håstad. On the approximation resistance of a random predicate. In APPROX-RANDOM, pages $149-163,2007.3$

[12] J. Håstad and A. Wigderson. Simple analysis of graph tests for linearity and PCP. Random Struct. Algorithms, 22(2):139-160, 2003. 3

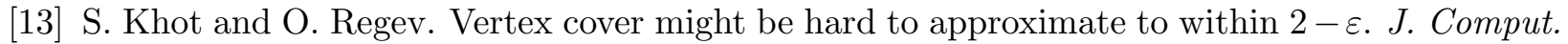
Syst. Sci., 74(3):335-349, 2008. 4

[14] K. Makarychev and Y. Makarychev. Personal communication. 2008. 1, 3, 15

[15] Y. Nesterov. Quality of semidefinite relaxation for nonconvex quadratic optimization. CORE Discussion Paper 9719, 1997. 1, 2, 7

[16] P. Raghavendra. Optimal algorithm and inapproximability results for every csp? In STOC : ACM Symposium on Theory of Computing, pages 245-254, 2008. 3

[17] A. Samorodnitsky and L. Trevisan. A PCP characterization of NP with optimal amortized query complexity. In STOC : ACM Symposium on Theory of Computing, pages 191-199, 2000. 3,10

[18] A. Samorodnitsky and L. Trevisan. Gowers uniformity, influence of variables, and PCPs. In STOC: ACM Symposium on Theory of Computing, pages 11-20, 2006. 1, 2, 3, 5, 10, 11

[19] L. Trevisan. Parallel approximation algorithms by positive linear programming. Algorithmica, 21(1):72-88, 1998. 3

[20] U. Zwick. Approximation algorithms for constraint satisfaction problems involving at most three variables per constraint. In Proceedings of the Ninth Annual ACM-SIAM Symposium on Discrete Algorithms, pages 201-210, 1998. 3 


\section{A Proof of Lemma 4.3}

Lemma. 4.3 Let $b_{0}, b_{1}, \ldots, b_{q-1}$ be real numbers such that $\sum_{j \in[q]} b_{j}=0$. For any $\delta>0$,

$$
\sum_{j \in[q]}(1+\delta)^{b_{j}} \geqslant \frac{\delta^{2}}{2 q(q-1)^{2}} \sum_{j \in[q]} b_{j}^{2}
$$

Proof. Without loss of generality, we can assume $b_{0} \geqslant b_{1} \geqslant \ldots, \geqslant b_{q-1}$. Let $b=\max _{j \in[q]}\left\{\left|b_{j}\right|\right\}$. Since $\sum_{j \in[q]} b_{j}=0$, we have $b_{0} \geqslant \frac{b}{q-1}$.

$$
\begin{aligned}
\sum_{j \in[q]}(1+\delta)^{b_{j}} & >(1+\delta)^{b_{0}} \\
& >\frac{\delta^{2}}{2} b_{0}^{2} \geqslant \frac{\delta^{2}}{2} \frac{b^{2}}{(q-1)^{2}} \\
& \geqslant \frac{\delta^{2}}{2 q(q-1)^{2}} \sum_{j \in[q]} b_{j}^{2}
\end{aligned}
$$

